\title{
Voluntary Physical Exercise Promotes Ocular Dominance Plasticity in Adult Mouse Primary Visual Cortex
}

\author{
Evgenia Kalogeraki, Franziska Greifzu, Franziska Haack, and Siegrid Löwel \\ Department of Systems Neuroscience, Johann-Friedrich-Blumenbach Institut für Zoologie und Anthropologie, and Bernstein Fokus Neurotechnologie, \\ Georg-August-Universität, 37075 Göttingen, Germany
}

Ocular dominance (OD) plasticity in the mouse primary visual cortex (V1) declines during aging and is absent beyond postnatal day (P) 110 when mice are raised in standard cages (SCs; Lehmann and Löwel, 2008). In contrast, raising mice in an enriched environment (EE) preserved a juvenile-like OD plasticity into late adulthood (Greifzu et al., 2014). EE raising provides the mice with more social interactions, voluntary physical exercise, and cognitive stimulation compared with SC, raising the question whether all components are needed or whether one of them is already sufficient to prolong plasticity. To test whether voluntary physical exercise alone already prolongs the sensitive phase for OD plasticity, we raised mice from $7 \mathrm{~d}$ before birth to adulthood in slightly larger than normal SCs with or without a running wheel (RW). When the mice were older than P135, we visualized V1 activity before and after monocular deprivation (MD) using intrinsic signal optical imaging. Adult RW-raised mice continued to show an OD shift toward the open eye after $7 \mathrm{~d}$ of MD, while age-matched SC mice without a RW did not show OD plasticity. Notably, running just during the $7 \mathrm{~d}$ MD period restored OD plasticity in adult SC-raised mice. In addition, the OD shift of the RW mice was mediated by a decrease of deprived-eye responses in V1, a signature of "juvenile-like" plasticity. We conclude that voluntary physical exercise alone is sufficient to promote plasticity in adult mouse V1.

Key words: adult plasticity; enriched environment; optical imaging; running

\section{Introduction}

One of the best studied models of experience-dependent plasticity is the ocular dominance (OD) plasticity in the primary visual cortex (V1). While neurons in the binocular part of V1 respond to inputs from both eyes, they are dominated by the contralateral eye in rodents. Occluding one eye for brief periods of time [monocular deprivation (MD)] induces a shift in the OD of binocular neurons toward the open eye (Wiesel and Hubel, 1963). OD plasticity in mice is age dependent: most pronounced in juvenile animals [postnatal day (P) 28], reduced in young adults (P90), and absent beyond P110 if mice are raised in standard cages (SCs; Espinosa and Stryker, 2012). Juvenile and adult OD plasticity are mechanistically different: in juveniles, the OD shift after $4 \mathrm{~d}$ of MD is mediated by a decrease of deprived-eye responses in V1 while in 3-monthold mice the OD shift is mediated by an increase of open-eye responses in $\mathrm{V} 1$ and $7 \mathrm{~d}$ of $\mathrm{MD}$ are needed for a significant shift (Sato and Stryker, 2008).

\footnotetext{
Received July 1, 2014; revised Sept. 18, 2014; accepted Oct. 7, 2014.

Author contributions: S.L. designed research; E.K., F.G., and F.H. performed research; E.K., F.G., and F.H. analyzed data; E.K., F.G., and S.L. wrote the paper.

This work was supported by German Federal Ministry of Education and Research Grants 01GQ0921 (F.G., E.K.) and $01 \mathrm{GQ0810} \mathrm{(S.L.)} \mathrm{and} \mathrm{by} \mathrm{grants} \mathrm{from} \mathrm{the} \mathrm{Deutsche} \mathrm{Forschungsgemeinschaft} \mathrm{through} \mathrm{the} \mathrm{Collaborative} \mathrm{Research} \mathrm{Center} 889$ (Cellular Mechanisms of Sensory Processing, Project B5; S.L.). We thank K.-F. Schmidt for help with initial experiments and M. Schink for excellent animal care.

The authors declare no competing financial interests.

This article is freely available online through the J Neurosci Author Open Choice option.

Correspondence should be addressed to Siegrid Löwel, Department of Systems Neuroscience, Johann-Friedrich-

Blumenbach-Institut für Zoologie und Anthropologie and Bernstein Fokus Neurotechnologie, Georg-AugustUniversität Göttingen, Von-Siebold-Str. 6, D-37075 Göttingen, Germany. E-mail: sloewel@gwdg.de.

DOI:10.1523/JNEUROSCI.2678-14.2014

Copyright $\odot 2014$ the authors $\quad 0270-6474 / 14 / 3315476-06 \$ 15.00 / 0$
}

Environmental enrichment (EE) accelerates the development of the visual system and enhances visual cortex plasticity in adult rats (Sale et al., 2007). Inspired by this finding, we showed that mice raised in EE preserved a juvenile-like OD plasticity into late adulthood (Greifzu et al., 2014). EE mice are housed in larger groups and bigger cages with a variety of environmental stimuli, such as running wheels, labyrinths, and tunnels. EE provides optimal conditions for enhanced exploration, social interaction, cognitive stimulation, and voluntary physical training (van Praag et al., 2000). The diversity of the EE components raises the question of the contribution of each of them in modulating plasticity. The different components of EE were already studied with respect to their role in recovery from amblyopia in rats (Baroncelli et al., 2012): physical exercise and visual enrichment promoted the recovery from amblyopia, whereas social interaction had no effect. Temporally coherent visual stimuli were also shown to increase OD plasticity in 3-month-old SC-raised mice (Matthies et al., 2013). Here, we focus on the effect of running on the timing of the sensitive phase for OD plasticity in mouse V1. Locomotion has recently been shown to massively increase pyramidal cell firing in V1: running caused a twofold to threefold increase in the sensory response of neurons in mouse V1 (Niell and Stryker, 2010; Fu et al., 2014) and promoted activity-dependent changes in V1 circuitry (Kaneko and Stryker, 2014). In the present study, we raised mice in SCs with or without a running wheel (RW) until the age of 4-5 months. V1 activity was then imaged before and after $7 \mathrm{~d}$ of MD using intrinsic signal optical imaging. In RW mice, but not in mice without a RW, OD plasticity was preserved up to at least P242 (oldest animal tested). In addition, running just during the $7 \mathrm{~d}$ MD period also promoted plasticity in adult 


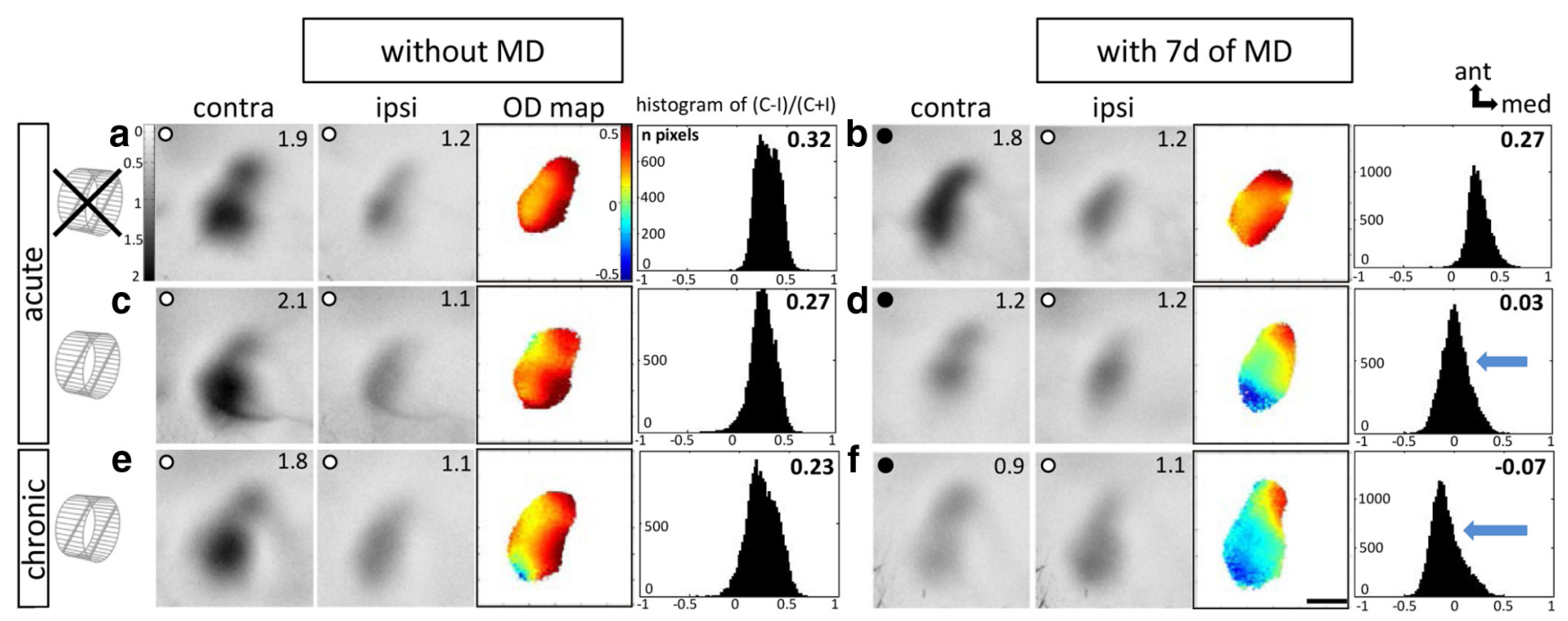

Figure 1. Running promoted OD plasticity in adult mouse V1. $a-f$, Optically recorded activity maps of the contralateral (contra) and ipsilateral (ipsi) eye in binocular V1 in mice raised in SCs with $(\boldsymbol{c}-\boldsymbol{f})$ or without $(\boldsymbol{a}, \boldsymbol{b})$ a RW, before $(\boldsymbol{a}, \boldsymbol{c}, \boldsymbol{e})$ and after $7 \mathrm{~d}$ of $\mathrm{MD}(\boldsymbol{b}, \boldsymbol{d}, \boldsymbol{f})$. Maps in $\boldsymbol{a}-\boldsymbol{d}$ are from acutely imaged mice. Maps in $\boldsymbol{e}$ and $\boldsymbol{f}$ are from a chronically imaged animal. Grayscale-coded response magnitude maps (numbers correspond to quantified V1 activation), two-dimensional OD maps, and the histogram of OD scores, including the average ODI, are illustrated. $\boldsymbol{a}, \boldsymbol{c}, \boldsymbol{e}$, Without MD, activity patches evoked by stimulation of the contralateral eye were darker than those of the ipsilateral eye, the average $0 \mathrm{DI}$ was positive, and warm colors prevailed in the $0 \mathrm{D}$ maps, indicating contralateral dominance. $\boldsymbol{b}, \boldsymbol{d}, \boldsymbol{f}$, While $7 \mathrm{~d}$ of MD did not induce OD plasticity in mice without a RW (b), it induced a strong OD shift toward the open eye in age-matched adult RW mice $(\boldsymbol{d}, \boldsymbol{f})$ : after MD, the contralateral and ipsilateral eye activated V1 with nearly equal strength, colder colors appeared in the OD map, and the histogram of OD scores shifted to the left (blue arrows). Scale bar, $1 \mathrm{~mm}$.

$(\leq \mathrm{P} 214)$ SC-raised mice. Notably, OD shifts were juvenile-like, i.e., mediated by a decrease of deprived-eye responses in V1. We conclude that voluntary running alone can promote a juvenilelike OD plasticity in adult V1.

\section{Materials and Methods}

Animals and rearing conditions

Long-term RW/no-RW group. Pregnant C57BL/6J females were put into slightly larger than normal SCs $(27 \times 43 \times 19 \mathrm{~cm})$ either with or without a RW 6-11 d before delivery. Offspring were separated into female and male groups at P28 without changing the housing conditions. Mice were housed with 3-5 mice per cage in eight cages as follows: two cages with male mice and no RWs; two cages with female mice and no RWs; two cages with male mice and RWs; two cages with female mice and RWs.

Short-term RW group. Mice were raised in SCs until at least P134 (4-5 animals per cage; only males), and transferred to SCs with a RW (size as above) only during the $7 \mathrm{~d} \mathrm{MD} /$ no-MD period.

The number of RW turns was counted daily and average RW turns per animal per day were calculated. All animals were at least P130 for MD. After MD/no-MD, spatial vision was checked daily by optomotry. Finally, we visualized V1 activation after stimulation of the ipsilateral and contralateral eye using intrinsic signal optical imaging. Imaging was always performed in the hemisphere contralateral to the deprived eye. All mice were from the mouse colony of the central animal facility of the University Medical Center Göttingen and housed with a $12 \mathrm{~h}$ light/dark cycle and with food and water available ad libitum. The experimental procedures comply with National Institutes of Health guidelines for the use of animals.

\section{Experimental groups}

Long-term RW/no-RW mice were separated into four groups: (1) with RW, without MD (RW-noMD; $n=7)$, (2) with RW and MD (RW + MD; $n=8$ ), (3) without RW, without MD (noRW-noMD; $n=5$ ), (4) without RW, with MD (noRW+MD; $n=5$ ). Short-term RW mice were separated into two groups: (1) with RW, without MD (7dRW-noMD; $n=6)$, (2) with RW and MD ( $7 \mathrm{dRW}+\mathrm{MD} ; n=6)$. The indicated age of mice is at day of the optical imaging experiment.

\section{Monocular deprivation}

The right eye was deprived for $7 \mathrm{~d}$ according to published protocols (Gordon and Stryker, 1996; Greifzu et al., 2014). After MD, the mice were returned to their home cages. Animals were checked daily to make sure that the eyes remained closed.

\section{Optomotry}

Both the spatial frequency threshold ("visual acuity") and the contrast threshold ("contrast sensitivity") of the optomotor reflex of all mice were measured using the optomotor system of Prusky et al. (2004) as described previously (Greifzu et al., 2014).

\section{Optical imaging of intrinsic signals and visual stimuli}

Surgery. Surgical preparations for optical imaging were performed as published (Kalatsky and Stryker, 2003; Greifzu et al., 2014). Briefly, mice were box-anesthetized with $2 \%$ halothane in $\mathrm{O}_{2}$ and $\mathrm{N}_{2} \mathrm{O}(1: 1)$ and injected with atropine $(0.3 \mathrm{mg} / \mathrm{mouse}$, s.c.; Franz Köhler Chemie), dexamethasone $(0.2$ $\mathrm{mg} / \mathrm{mouse}$ s.c.; Ratiopharm), and chlorprothixene ( $0.2 \mathrm{mg} / \mathrm{mouse}$, i.m.; Sigma-Aldrich). After placing animals in a stereotaxic frame, anesthesia was maintained with $0.8 \%$ halothane in a 1:1 mixture of $\mathrm{O}_{2}$ and $\mathrm{N}_{2} \mathrm{O}$.

Data acquisition and visual stimulation. Mouse V1 responses were recorded through the skull using the "Fourier" imaging method of Kalatsky and Stryker (2003) and optimized for the assessment of OD plasticity (Cang et al., 2005a). V1 signals were visualized with a CCD camera (Dalsa $1 \mathrm{M} 30$ ) using a $135 \times 50 \mathrm{~mm}$ tandem lens configuration (Nikon) with red illumination light $(610 \pm 10 \mathrm{~nm})$. Active brain regions absorb more of the red light and appear darker in the images. Frames were acquired at a rate of $30 \mathrm{~Hz}$, temporally binned to $7.5 \mathrm{~Hz}$, and stored as $512 \times 512$-pixel images after spatial binning of the camera image.

Visual stimuli were presented on a high refresh rate monitor (21 inches; Accuvue HM-4921-D, Hitachi) positioned $25 \mathrm{~cm}$ from the eyes. Stimuli consisted of white drifting horizontal bars $\left(2^{\circ}\right.$ wide) as described previously (Greifzu et al., 2014). The amplitude component of the optical signal represents the intensity of neuronal activation (expressed as fractional change in reflectance times $10^{-4}$ ) and was used to calculate OD. At least three maps per animal were averaged to compute the OD index (ODI) as $(C-I) /(C+I)$, with $C$ and $I$ representing the response magnitudes of each pixel to visual stimulation of the contralateral $(C)$ and ipsilateral ( $I$ ) eye. OD maps were assessed by an experimenter blind to the animals' rearing conditions.

To determine $\mathrm{V} 1$ activation and the quality of the retinotopic maps, we used full-field $\left(78^{\circ} \times 59^{\circ}\right)$ horizontal/vertical moving bars (elevation/ azimuth maps) presented in the right visual field to the right eye. The 
quality of retinotopic maps was assessed as the SD of the pixel position differences (Cang et al., 2005b) with lower values indicating higher map quality.

\section{Statistical analysis}

All intragroup and intergroup comparisons were analyzed by a twotailed Student's $t$ test (with Bonferroni's correction). The intergroup comparison of the enhancement of visual acuity was analyzed by ANOVA with repeated measurements and Bonferroni correction. The levels of significance were set as ${ }^{\star} p<0.05,{ }^{* *} p<0.01$, and ${ }^{* * *} p<0.001$. Data are represented as means \pm SEM.

\section{Results}

Voluntary running prolonged the sensitive phase for OD plasticity in V1 into adulthood

In both mice raised with a RW and mice raised without a RW, V1 activation in the binocular zone was dominated by the contralateral eye: activity patches induced by visual stimulation of the contralateral eye were always darker than those after ipsilateral eye stimulation, the average ODI was positive, and warm colors prevailed in the two-dimensional OD maps (Fig. 1a,c,e). Thus running per se did not change the basic OD distribution in V1. After $7 \mathrm{~d}$ of MD, the OD shifted toward the open eye only in the adult RW mice (Fig. $1 d, f$ ) while V1 activation of no-RW mice remained dominated by the contralateral (deprived) eye (Fig. $1 b)$ : in RW mice, the activity spot induced by stimulation of the open (ipsilateral) eye was as dark as the spot induced by stimulation of the deprived eye, colder colors predominated in the OD map, and the ODI histogram was shifted to the left (Fig. 1d). Qualitative and quantitative analyses of V1 activation indicated that the OD shift was mediated by a reduction of deprived-eye responses in V1 (Fig. 1c,d). To unambiguously determine the mechanism underlying the prolonged OD plasticity in adult RW mice, we additionally performed chronic imaging experiments before and after MD. In a first imaging session, V1 activities after visual stimulation of the ipsilateral and contralateral eye were determined, and then the right eye was deprived of vision for $7 \mathrm{~d}$. During MD, spatial vision was checked daily by optomotry. After the MD period, we imaged V1 activities again (second imaging) and quantitatively compared values before and after deprivation. Activity maps recorded before MD were dominated by input from the contralateral eye, warm colors prevailed in the OD map, and the average ODI was positive (Fig. 1e). After $7 \mathrm{~d}$ of $\mathrm{MD}, \mathrm{V} 1$ activation via the contralateral eye was visibly reduced so that both eyes activated V1 with approximately the same strength, colder colors prevailed in the OD map, ODI values were reduced, and the ODI histogram was shifted to the left (Fig. 1f).

Quantitative analyses of V1 activation for all recorded maps (acute and chronic) showed that the average ODI of RW mice older than P138 decreased from $0.26 \pm 0.03(n=7, \mathrm{P} 140-\mathrm{P} 240)$ without $\mathrm{MD}$ to $0.06 \pm 0.03(n=8, \mathrm{P} 138-\mathrm{P} 242)$ with $\mathrm{MD}$ (Bonferroni-adjusted $t$ test, $p=0.0003$ ). In contrast, adult no-RW mice did not display OD plasticity (ODI: no MD, $0.32 \pm$ $0.02, n=5, \mathrm{P} 137-\mathrm{P} 200 ; \mathrm{MD}, 0.25 \pm 0.03, n=5, \mathrm{P} 145-\mathrm{P} 195 ; p=$ 0.1126 , Bonferroni-adjusted $t$ test; Fig. $2 a$ ). ODIs after MD were different between RW mice and no-RW mice ( $p=0.0009$, Bonferroni-adjusted $t$ test).

Quantification further showed that the OD shift of RW mice was mediated by a decrease of deprived-eye responses in V1: V1 activity after stimulating the deprived (contralateral) eye was $1.59 \pm 0.10$ without $\mathrm{MD}(n=7)$ and $1.18 \pm 0.09$ with $\mathrm{MD}(n=$ $8 ; p=0.008, t$ test). In contrast, open (ipsilateral) eye responses of RW mice remained unchanged (no MD: $0.95 \pm 0.06, n=7$; MD: $1.13 \pm 0.09, n=8 ; p=0.143$, $t$ test; Fig. $2 b$ ). As expected from the absent OD shift in no-RW mice, V1 activation after stimulating the ipsilateral and contralateral eye did not change after $\mathrm{MD}$, and V1 remained dominated by the contralateral eye, despite the modified visual input (contralateral eye: no MD, $1.60 \pm 0.09$, $n=5 ; \mathrm{MD}, 1.67 \pm 0.07, n=5 ; p=0.525, t$ test; ipsilateral eye: no MD: $0.88 \pm 0.09, n=5 ; \mathrm{MD}, 1.11 \pm 0.11, n=5 ; p=0.134$, $t$ test; Fig. $2 b)$.

To test whether long-term running is required for prolonging OD plasticity or whether running can promote plasticity in adult V1 also "acutely," we raised another group of mice in SCs until P134 and transferred them to SCs with a RW only during $7 \mathrm{~d}$ $\mathrm{MD} /$ no-MD period. While the average ODI for $7 \mathrm{~d} \mathrm{RW}$ mice without $\mathrm{MD}$ was $0.21 \pm 0.01(n=6, \mathrm{P} 143-\mathrm{P} 217)$, it decreased to $0.06 \pm 0.01$ after MD (7dRW+MD: $n=6$, P141-P214; compared with 7dRW-noMD, $p=0.0001$; noRW + MD, $0.0001 ; t$ test; Fig. $2 c$ ). The running-enabled OD shift was again mediated by decreased deprived-eye responses in V1: V1 activation through the contralateral eye was reduced from $2.00 \pm 0.08(n=$ $6)$ to $1.34 \pm 0.22$ after $\mathrm{MD}(n=6, p=0.037, t$ test $)$, whereas ipsilateral eye $\mathrm{V} 1$ responses did not change (no MD: $1.40 \pm 0.10$, $n=6$; MD: $1.35 \pm 0.13, n=6 ; p=0.77$, $t$ test; Fig. $2 d$ ).

\section{Retinotopic maps and V1 activation were similar in RW and no-RW mice}

The retinotopic and activity maps were compared in RW $(n=7)$, no-RW $(n=5)$, and $7 \mathrm{~d}$ RW $(\mathrm{n}=6)$ mice. For all three "raising" conditions, elevation and azimuth maps were nearly identical in signal strength and retinotopic map quality. The magnitude of the $\mathrm{V} 1$ responses after elevation stimulation was $2.78 \pm 0.33$ for RW mice, $2.46 \pm 0.15$ for no-RW mice, and $3.10 \pm 0.33$ for $7 \mathrm{~d}$ RW mice ( $p=0.17$, ANOVA). Likewise, azimuth maps were similar (RW, $2.27 \pm 0.22$; no-RW, $2.18 \pm 0.15$; 7 d RW, $2.70 \pm$ $0.24 ; p=0.26$, ANOVA), as was the quality of the retinotopic maps (elevation: RW, $1.5 \pm 0.4$; no-RW, $1.8 \pm 0.5$; $7 \mathrm{~d}$ RW, $1.3 \pm$ $0.5 ; p=0.36$, ANOVA; azimuth: RW, $4.8 \pm 2.0$; no-RW, $2.8 \pm$ 1.0; 7 d RW, $2.6 \pm 0.7 ; p=0.43$, ANOVA).

\section{Basic visual abilities and enhanced optomotor reflex after MD were not different between mice with and without a RW}

Spatial vision of all experimental mice was measured using the virtual-reality optomotor setup (Prusky et al., 2004) before and after MD. The highest spatial frequency gratings that elicited an optomotor reflex (visual acuity) was $0.37 \pm 0.003$ cycles $/{ }^{\circ}$ in RW mice $(n=15)$ and $0.37 \pm 0.002$ cycles $/{ }^{\circ}$ in no-RW mice $(n=10)$, and thus not different between the groups $(p=0.901$, Bonferroni-adjusted $t$ test). Values were also similar to previously published values for SC-raised BL/6 mice (Prusky et al., 2004; Lehmann and Löwel, 2008). Likewise, baseline contrast sensitivity values were not different between RW and no-RW mice (contrast sensitivity at 0.031 cycles $/^{\circ}: \mathrm{RW}, 4 \pm 0.1$; no-RW, $4 \pm 0.01$; contrast sensitivity at 0.064 cycles $/{ }^{\circ}: \mathrm{RW}, 14 \pm 0.2$; no-RW, $14 \pm$ 0.1 ; contrast sensitivity at $0.092 \mathrm{cycle}^{\circ}{ }^{\circ}: \mathrm{RW}, 13 \pm 0.1$; no-RW, $13 \pm 0.1$; contrast sensitivity at $0.103 \mathrm{cycle}^{\circ}$ : RW, $12 \pm 0.1$; no-RW, $12 \pm 0.1$; contrast sensitivity at $0.192 \mathrm{cycles}^{\circ}{ }^{\circ}: \mathrm{RW}, 7 \pm$ 0.1 ; no-RW, $7 \pm 0.1$; contrast sensitivity at 0.272 cycles $^{\circ}$ : RW, $4 \pm 0.1$; no-RW, $4 \pm 0.01 ; p>0.05$ for every spatial frequency, ANOVA). Thus, daily voluntary physical exercise did not change basic spatial vision, at least up to P242.

After MD and daily testing in the optomotor setup, visual acuity and contrast sensitivity values of the optomotor reflex through the open eye increased similarly in both groups. Visual acuity increased on average by $29 \%$ to $0.48 \pm 0.04$ cycles $/{ }^{\circ}$ after $\mathrm{MD}(n=8, p<0.001$ Bonferroni-adjusted $t$ test $)$ in RW mice and 

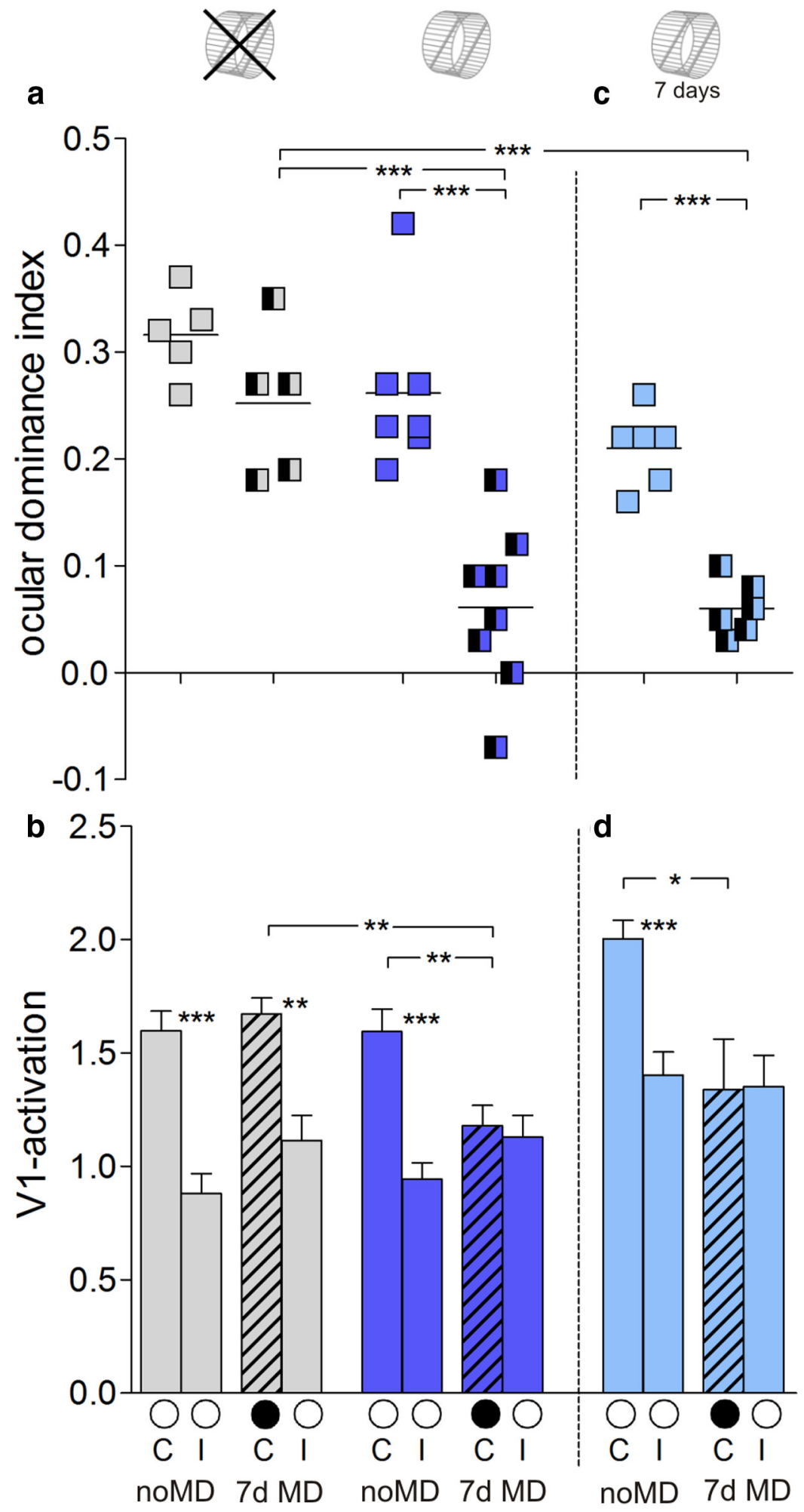

Figure 2. Quantification of V1 activation with and without a RW. a, c, Optically imaged ODIs of control animals and after MD in no-RW mice (gray), RW mice (blue), and $7 \mathrm{~d}$ RW mice (light blue). Symbols represent ODI values of individuals. Means are marked by horizontal lines. $\boldsymbol{b}, \boldsymbol{d}, \mathrm{V} 1$ activation elicited by stimulation of the contralateral (C) or ipsilateral (I) eye in control animals and after MD (black filled circle indicates MD eye). ${ }^{*} p<0.05,{ }^{* *} p<0.01$, and ${ }^{* * *} p<0.001$.

by $34 \%$ to $0.50 \pm 0.03$ cycles $/{ }^{\circ}$ after MD in no-RW mice $(n=5$, $p<0.001$, Bonferroni-adjusted $t$ test). For the $7 \mathrm{~d}$ RW group, visual acuity increased on average by $27 \%$ from $0.37 \pm 0.01 \mathrm{cy}-$ cles ${ }^{\circ}$ before MD to $0.47 \pm 0.02$ cycles $/{ }^{\circ}$ after $\mathrm{MD}(n=6, p<$ 0.001 , Bonferroni-adjusted $t$ test; Fig. 3). The increase in visual acuity was not different between the groups ( $p>0.05$, Bonferroni-adjusted $t$ test). Likewise, contrast sensitivity thresholds of the optomotor reflex of the open eye also increased after MD in RW mice (on day 7 after MD, compared with values from day $0: 0.031 \mathrm{cycles} /{ }^{\circ}$, to $6 \pm 0.4, p>$ $0.05 ; 0.064$ cycles $/^{\circ}$, to $28 \pm 4, p<0.001$; 0.092 cycles $/{ }^{\circ}$, to $29 \pm 5, p<0.001 ; 0.103$ cycles $^{\circ}$, to $32 \pm 8, p<0.001 ; 0.192 \mathrm{cy}$ cles $/^{\circ}$, to $22 \pm 7, p<0.01 ; 0.272$ cycles $/{ }^{\circ}$, to $9 \pm 4, p>0.01$, ANOVA). This increase after MD was similar to no-RW mice $\left(0.031\right.$ cycles $/{ }^{\circ}, 7 \pm 0.4 ; 0.064$ cycles $/{ }^{\circ}$, $30 \pm 3.4 ; 0.092$ cycles $^{\circ}, 36 \pm 4.6 ; 0.103$ cycles ${ }^{\circ}, 37 \pm 5.2 ; 0.192$ cycles $/^{\circ}, 25 \pm 3.4$; 0.272 cycles $/{ }^{\circ}, 7 \pm 0.8 ; p>0.05$ for every frequency comparison between RW and no-RW groups, ANOVA). Mice without MD (including both RW and no-RW mice) did not show any change in visual acuity or contrast sensitivity over days.

To summarize, neither basic spatial vision nor the experience-enabled increase of thresholds after MD were modified by RW experience during adolescence.

\section{Amount of running and body weight}

The average amount of RW turns for male RW mice was $3229 \pm 212$ per day, corresponding to $1.28 \pm 0.09 \mathrm{~km}$; and $3991 \pm$ 445 turns/d for female RW mice, corresponding to $1.60 \pm 0.18 \mathrm{~km}$. The difference in RW turns between males and females was not significant $(p>0.05, t$ test). For $7 \mathrm{~d}$ RW mice, the average number of RW turns/d was $3452 \pm 626$, corresponding to $1.38 \pm 0.25 \mathrm{~km}$, similar to the other groups $(p>0.05$, ANOVA). For both male and female mice, the average body weight before the final optical imaging experiment was similar in RW, noRW, and $7 \mathrm{~d}$ RW animals (males: RW, $26.6 \pm 0.8 \mathrm{~g}, n=8$; no-RW, $27.9 \pm 0.6 \mathrm{~g}$, $n=4 ; 7 \mathrm{~d} \mathrm{RW}, 29.6 \pm 0.3 \mathrm{~g}, n=12, p=$ 0.46 , ANOVA; females: RW, $22.2 \pm 0.6 \mathrm{~g}$, $n=7$; no-RW, $20.8 \pm 0.2 \mathrm{~g}, n=4, p=$ $0.1117, t$ test).

There were no sex differences for ODI, V1 activation, or map quality ( $p>0.05$ for each parameter checked, $t$ test).

\section{Discussion}

Our results provide clear evidence that raising mice in SCs equipped with a RW for voluntary physical exercise prolonged the sensitive phase for OD plasticity into late adulthood. Actually, the oldest mouse raised with a RW was already $242 \mathrm{~d}$ old and thus way beyond the sensitive phase for OD plasticity for SC-raised mice (Lehmann and Löwel, 2008). This mouse still showed a very strong OD shift toward the open eye after MD. In contrast, in mice raised without a RW, OD plasticity ceased after 


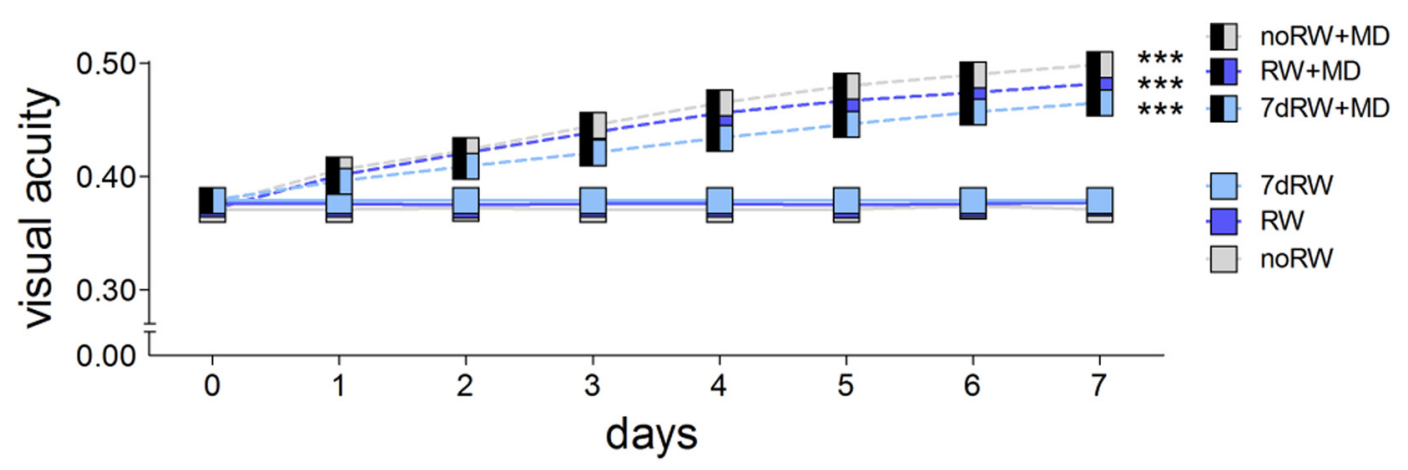

Figure 3. Running had no effect on the optomotor reflex and its enhancement after MD. Visual acuity of the optomotor reflex plotted against days after MD. ${ }^{* *} p<0.001$.

P110. Notably, running only during MD had essentially the same effect on OD plasticity as long-term running. In addition, the preserved OD shift in the RW mice was predominantly mediated by a reduction of deprived-eye responses in $\mathrm{V} 1$, a typical signature of juvenile-like plasticity. Thus, life-long daily RW running in mice seems to preserve a juvenile brain and exert similar effects on prolonging visual cortical plasticity as the more complex EE raising. Furthermore, even short-term running can restore OD plasticity in adult V1.

It is widely recognized that $\mathrm{EE}$ has a positive effect on neuronal plasticity. EE usually provides a combination of motor, social, and cognitive stimulating factors (Sale et al., 2014). In an attempt to disentangle the roles of the various factors, it was recently shown that both physical exercise and visual enrichment promoted the recovery from long-term amblyopia in rat V1, whereas social interaction had no effect (Baroncelli et al., 2012). It had been shown before that social grouping alone is insufficient to explain the cerebral effects of EE in rats (Rosenzweig et al., 1978). In humans, improvement in amblyopic vision was achieved by visual enrichment with video games (Bavelier et al., 2010). Forced visual exposure to temporally coherent visual stimuli for $6 \mathrm{~h} / \mathrm{d}$ was also recently shown to increase OD plasticity in 3-month-old SC-raised mice (Matthies et al., 2013). Moreover, enhanced sensory and motor input in EE promoted physiological maturation and consolidation of visual cortical circuits in dark-reared rats and thus even without visual experience (Bartoletti et al., 2004). However, it was not yet investigated whether physical exercise alone can alter the timing of the sensitive phase for OD plasticity. Physical exercise is a very interesting parameter since locomotion causes a twofold to threefold increase in the sensory response of neurons in mouse V1 (Niell and Stryker, 2010; Fu et al., 2014; Wilson and Glickfeld, 2014) and also enhances visual performance (Bennett et al., 2013). Enhanced visual processing might in turn, for example via direct anatomical connections (Di Garbo et al., 2011) or nicotinic inputs from the basal forebrain (Fu et al., 2014), promote plasticity in V1.

While voluntary physical exercise seems to basically mimic major effects of general EE on OD plasticity, the OD shift of the RW mice was nevertheless not as pronounced as that observed in adult mice raised in a complex EE (Greifzu et al., 2014). Based on these observations we conclude that running alone can already prolong OD plasticity in mice, but additional EE components can increase the plasticity-promoting effect.

Most interestingly, short-term running had essentially the same effect on OD plasticity as long-term running: up to an age of $\geq \mathrm{P} 214,7 \mathrm{~d}$ of running during MD was sufficient to restore a juvenile-like OD plasticity in SC-raised mice. This observation complements a recent study showing that $4 \mathrm{~h}$ of running with visual stimulation promoted recovery from long-term MD up to P180 (Kaneko and Stryker, 2014). While recovery of V1 responses was specific to the particular visual stimuli presented during locomotion, in our study, no particular visual stimulus was presented during running.

Interestingly, the OD shift in both groups of RW mice was predominantly mediated by reductions in deprived-eye responses in V1. Those reductions were previously only observed after $4 \mathrm{~d}$ of MD in 4-week-old mice (Hofer et al., 2006; Heimel et al., 2007; Sato and Stryker, 2008) and after $7 \mathrm{~d}$ of MD in adult mice raised in an EE (Greifzu et al., 2014). It is interesting to note that $\mathrm{EE}$ raising also preserved a juvenile GABA/AMPA ratio into adulthood (Greifzu et al., 2014) and that running has been shown to act predominantly via disinhibitory circuits, which reduce inhibition on layer $2 / 3$ pyramidal neurons (Fu et al., 2014). In fact, it was shown that providing adult rats with RWs for 3 weeks caused a decrease in GABA release (Baroncelli et al., 2012). Thus, extended running experience by raising mice in SCs with RWs or in EE cages containing RWs may "chronically" reduce inhibitory drive onto pyramidal cells and thus promote cortical plasticity. However, it is likely that other molecules and mechanisms that are known to be affected by EE housing and involved in regulating OD plasticity are also involved in the effect of running, e.g., brain-derived neurotrophic factor, perineuronal nets, insulinlike growth factor I, serotonin, and histone acetylation (for review, see Sale et al., 2014). While running alone is sufficient to preserve a juvenile-like OD plasticity in V1, and thus presumably a juvenile brain into late adulthood, other components of complex EE are likely to intensify this effect. Short-term running may enhance cortical function and plasticity via activation of neuromodulatory circuits (Carcea and Froemke, 2013) and additionally may induce hemodynamic changes that modify cortical circuit function (Seifert and Secher, 2011).

We also tested baseline visual abilities and their experiencedependent increase after MD in the visual optomotor setup. There was no significant difference between RW mice, no-RW mice, and $7 \mathrm{~d}$ RW mice, and values were comparable to previously published data of SC-raised C57BL/6 mice (Prusky et al., 2006). This indicates that physical exercise had no effect on the spatial frequency and contrast thresholds of the optomotor reflex. Moreover, activation strength and quality of the retinotopic maps in V1 was indistinguishable between the RW and no-RW mice, indicating that these parameters were also not affected by physical exercise.

In summary, we conclude that physical exercise alone is not only capable of extending the sensitive phase for OD plasticity into late adulthood, and possibly lifelong in mice, but can also restore OD plasticity in adult SC-raised animals. While further 
research is needed to understand the cellular mechanisms underlying this effect, the present data already emphasize the power of exercise for maintaining plasticity into older age.

\section{References}

Baroncelli L, Bonaccorsi J, Milanese M, Bonifacino T, Giribaldi F, Manno I, Cenni MC, Berardi N, Bonanno G, Maffei L, Sale A (2012) Enriched experience and recovery from amblyopia in adult rats: impact of motor, social and sensory components. Neuropharmacology 62:2388-2397. CrossRef Medline

Bartoletti A, Medini P, Berardi N, Maffei L (2004) Environmental enrichment prevents effects of dark-rearing in the rat visual cortex. Nat Neurosci 7:215-216. CrossRef Medline

Bavelier D, Levi DM, Li RW, Dan Y, Hensch TK (2010) Removing brakes on adult brain plasticity: from molecular to behavioral interventions. J Neurosci 30:14964-14971. CrossRef Medline

Bennett C, Arroyo S, Hestrin S (2013) Subthreshold mechanisms underlying state-dependent modulation of visual responses. Neuron 80:350 -357. CrossRef Medline

Cang J, Kalatsky VA, Löwel S, Stryker MP (2005a) Optical imaging of the intrinsic signal as a measure of cortical plasticity in the mouse. Vis Neurosci 22:685-691. Medline

Cang J, Rentería RC, Kaneko M, Liu X, Copenhagen DR, Stryker MP (2005b) Development of precise maps in visual cortex requires patterned spontaneous activity in the retina. Neuron 48:797-809. CrossRef Medline

Carcea I, Froemke RC (2013) Cortical plasticity, excitatory-inhibitory balance, and sensory perception. Prog Brain Res 207:65-90. CrossRef Medline

Di Garbo A, Mainardi M, Chillemi S, Maffei L, Caleo M (2011) Environmental enrichment modulates cortico-cortical interactions in the mouse. PLoS One 6:e25285. CrossRef Medline

Espinosa JS, Stryker MP (2012) Development and plasticity of the primary visual cortex. Neuron 75:230-249. CrossRef Medline

Fu Y, Tucciarone JM, Espinosa JS, Sheng N, Darcy DP, Nicoll RA, Huang ZJ, Stryker MP (2014) A cortical circuit for gain control by behavioral state. Cell 156:1139-1152. CrossRef Medline

Gordon JA, Stryker MP (1996) Experience-dependent plasticity of binocular responses in the primary visual cortex of the mouse. J Neurosci 16: 3274-3286. Medline

Greifzu F, Pielecka-Fortuna J, Kalogeraki E, Krempler K, Favaro PD, Schlüter OM, Löwel S (2014) Environmental enrichment extends ocular dominance plasticity into adulthood and protects from stroke-induced impairments of plasticity. Proc Natl Acad Sci U S A 111:1150-1155. CrossRef Medline

Heimel JA, Hartman RJ, Hermans JM, Levelt CN (2007) Screening mouse vision with intrinsic signal optical imaging. Eur J Neurosci 25:795-804. CrossRef Medline

Hofer SB, Mrsic-Flogel TD, Bonhoeffer T, Hübener M (2006) Prior experience enhances plasticity in adult visual cortex. Nat Neurosci 9:127-132. CrossRef Medline

Kalatsky VA, Stryker MP (2003) New paradigm for optical imaging: temporally encoded maps of intrinsic signal. Neuron 38:529-545. CrossRef Medline

Kaneko M, Stryker MP (2014) Sensory experience during locomotion promotes recovery of function in adult visual cortex. eLife 3:e02798. CrossRef Medline

Lehmann K, Löwel S (2008) Age-dependent ocular dominance plasticity in adult mice. Plos One 3:e3120. CrossRef Medline

Matthies U, Balog J, Lehmann K (2013) Temporally coherent visual stimuli boost ocular dominance plasticity. J Neurosci 33:11774-11778. CrossRef Medline

Niell CM, Stryker MP (2010) Modulation of visual responses by behavioral state in mouse visual cortex. Neuron 65:472-479. CrossRef Medline

Prusky GT, Alam NM, Beekman S, Douglas RM (2004) Rapid quantification of adult and developing mouse spatial vision using a virtual optomotor system. Invest Ophthalmol Vis Sci 45:4611-4616. CrossRef Medline

Prusky GT, Alam NM, Douglas RM (2006) Enhancement of vision by monocular deprivation in adult mice. J Neurosci 26:11554-11561. CrossRef Medline

Rosenzweig MR, Bennett EL, Hebert M, Morimoto H (1978) Social grouping cannot account for cerebral effects of enriched environments. Brain Res 153:563-576. CrossRef Medline

Sale A, Maya Vetencourt JF, Medini P, Cenni MC, Baroncelli L, De Pasquale R, Maffei L (2007) Environmental enrichment in adulthood promotes amblyopia recovery through a reduction of intracortical inhibition. Nat Neurosci 10:679-681. CrossRef Medline

Sale A, Berardi N, Maffei L (2014) Environment and brain plasticity: towards an endogenous pharmacotherapy. Physiol Rev 94:189-234. CrossRef Medline

Sato M, Stryker MP (2008) Distinctive features of adult ocular dominance plasticity. J Neurosci 28:10278-10286. CrossRef Medline

Seifert T, Secher NH (2011) Sympathetic influence on cerebral blood flow and metabolism during exercise in humans. Prog Neurobiol 95:406-426. CrossRef Medline

van Praag H, Kempermann G, Gage FH (2000) Neural consequences of environmental enrichment. Nat Rev Neurosci 1:191-198. CrossRef Medline

Wiesel TN, Hubel DH (1963) Single-cell responses in striate cortex of kittens deprived of vision in 1 eye. J Neurophysiol 26:1003-1017. Medline

Wilson AM, Glickfeld LL (2014) Visual circuits get the VIP treatment. Cell 156:1123-1124. CrossRef Medline 